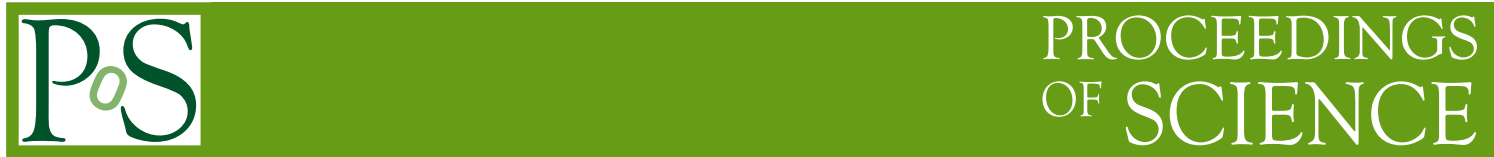

\title{
The Intensity Frontier at Fermilab
}

\section{Eric PREBYS*}

Fermi National Accelerator Laboratory

E-mail: prebysefnal.gov

As the highest energy collider in the world, the Tevatron has been the centerpiece of Fermilab's physics program for more than 20 years. This will all change with the imminent startup of CERN's LHC.

This note describes the lab's plans to redefine its mission in terms of the "intensity frontier", with a program focusing on neutrino physics, precision measurements and cutting edge accelerator R\&D. The key component of this plan is an intense new proton source, referred to as "Project $X$ ". Project $X$ and its associated physics program will be described and compared to related programs worldwide. Also discussed will be intermediate plans which will support that lab's physics programs while Project $\mathrm{X}$ is being designed and constructed.

European Physical Society Europhysics Conference on High Energy Physics

July 16-22, 2009

Krakow, Poland

\footnotetext{
${ }^{*}$ Speaker.

${ }^{\dagger}$ Representing the Fermi National Accelerator Laboratory.
} 


\section{Present Operation}

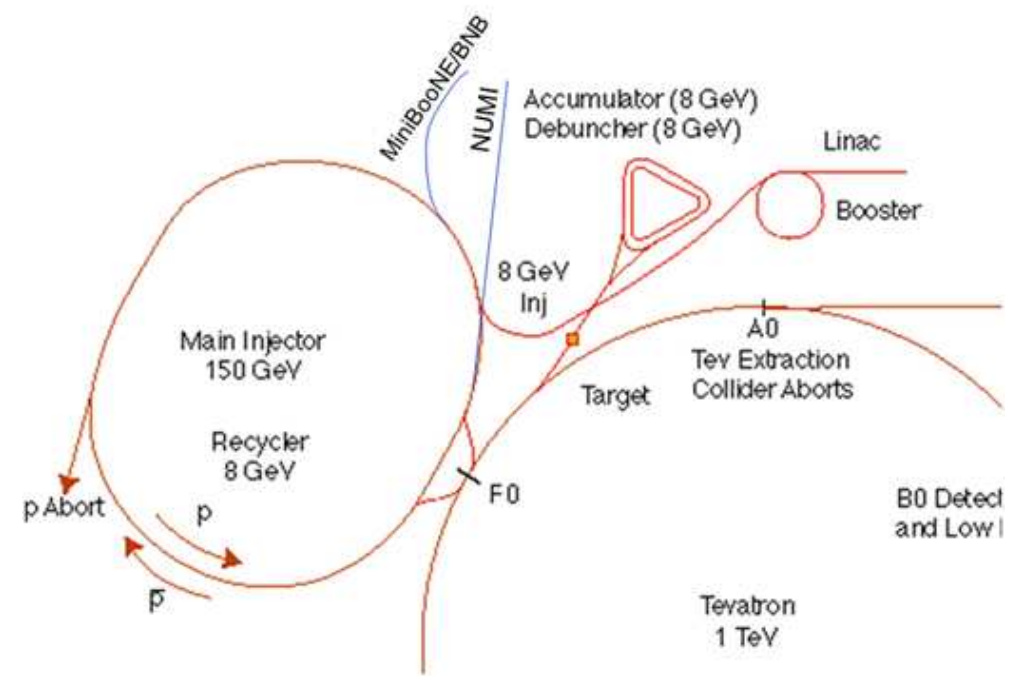

Figure 1: The Fermilab accelerator complex. The new Project $X$ accelerator will inject directly into the Recycler, eliminating the need for the aging Linac/Booster.

The Fermilab accelerator complex is shown in Figure 1. During collider operation, the Linac and Booster accelerate protons to $8 \mathrm{GeV}$ and inject them into the Main Injector, which accelerates them to $120 \mathrm{GeV}$ and delivers them to the antiproton production target. The antiprotons produced at this target are debunched, stochastically cooled, and collected in the Debuncher/Accumulator ring system. From there, antiprotons are transfered to the Recycler ring, an $8 \mathrm{GeV}$ storage ring made out of permanent magnets, which shares the tunnel with the Main Injector. When enough antiprotons have been produced, then both protons and antiprotons are accelerated to $150 \mathrm{GeV}$ by the Main Injector and loaded into the Tevatron in opposite directions. The Tevatron accelerates them to $980 \mathrm{GeV} /$ beam and initiates collisions. While they are colliding, the anti-proton production process continues. This "stack and store" cycle takes roughly a day.

During collider operation, the complex also supports a neutrino program. Protons for the MINOS experiment[1] are accelerated to $120 \mathrm{GeV}$ along with the protons for antiproton production. While the Main Injector is accelerating, $8 \mathrm{GeV}$ protons are sent directly from the Booster for the MiniBooNE experiment[2].

\section{Intensity Frontier}

The "Intensity Frontier" was identified by the Particle Physics Project Prioritization Panel (P5) as one of three important and related fields of fundamental physics research, the others being the "Energy Frontier" and the "Cosmic Frontier"[3].

The Intensity Frontier involves the use of Megawatt scale beams with energies from a few to a few hundred $\mathrm{GeV}$ for research into three key areas:

Neutrino Physics 
There are still very important unanswered questions about the nature of neutrinos. These include the mass hierarchy, absolute mass, exact mixing angles, CP-violation, and whether neutrinos are Dirac or Majorana. The NOvA experiment[4] is currently being constructed to answer some of these questions, using neutrino beam produced at Fermilab and detected near the Canadian border.

A next generation, longer baseline experiment is also being proposed for the new Deep Underground Science and Engineering Laboratory (DUSEL)[5]. This will also rely on a beam from Fermilab.

\section{Precision Measurements}

The search for rare decays and other precision measurements can probe mass scales well beyond those available to direct searches.

The proposed Mu2e experiment[6] will search for the conversion of a muon to an electron in the field of a nucleus. This experiment has become an important part of Fermilab's medium term plans.

Other precision experiments which are being seriously considered include a rare kaon decay program[7] and an improved measurement of g-2[8].

\section{Advanced Accelerator $R \& D$}

Intense proton beams can be used for important research into advanced accelerator concepts. In particular, they can be used to produce muons for a neutrino factory or muon collider.

There are also proposals to use intense proton beams to drive sub-critical nuclear reactors.

\section{Project X}

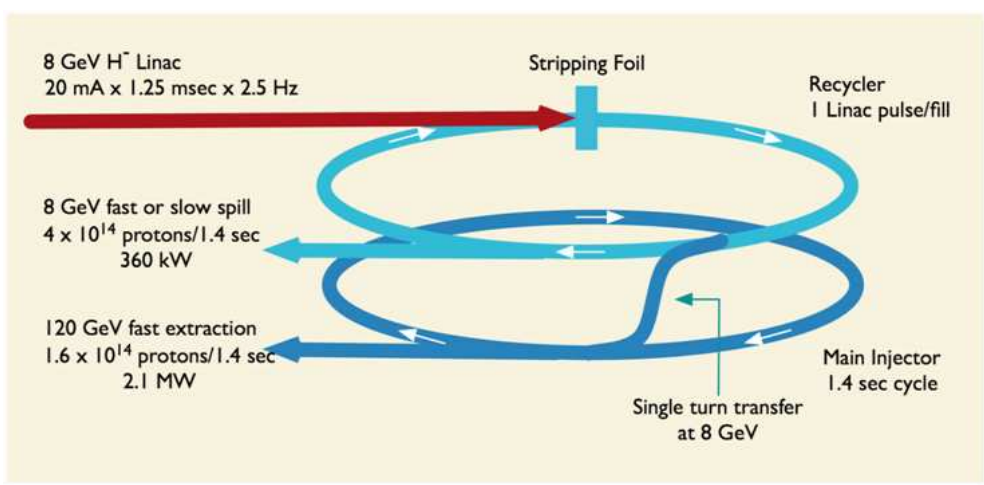

Figure 2: Schematic representation of Project X. Hydrogen ions are injected into the Recycler, where the electrons are stripped. One pulse is sent every 1.4 seconds to the Main Injector. While it is ramping up and down, additional linac pulses are available for other physics programs.

The total proton flux at Fermilab is limited by the capabilities of the existing Linac and Booster, which date back to the beginning of the lab. The lab's future plans center around "Project X"[9]: a new, intense proton source which will inject directly into the Recycler, which is currently used to store antiprotons. One pulse would supply enough protons, leaving additional pulses available for an $8 \mathrm{GeV}$ program, as illustrated in Figure 2.

In the base line configuration, the high energy portion is based on RF cavities and cryomodules designed for the ILC. The preceding section is similar, but with slightly compressed cavities to 


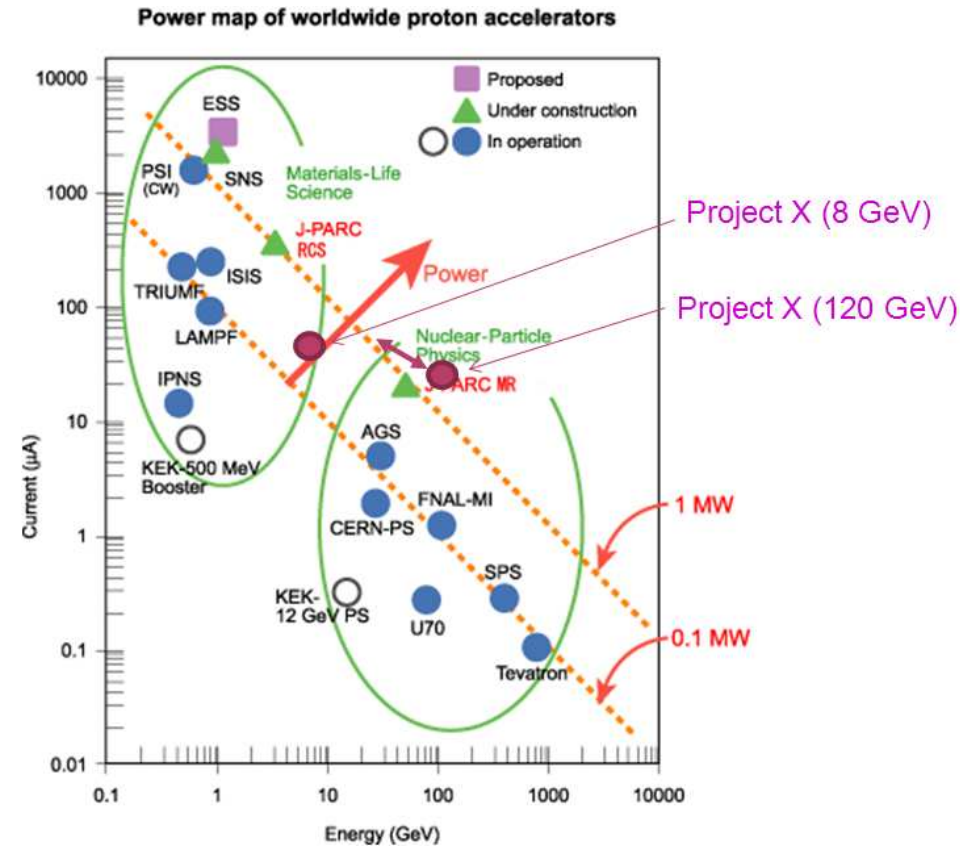

Figure 3: Available proton flux during the Project $X$ era is show in the context of of program around the world.

accommodate the lower $\beta$. A new ion source will feed energy section based on superconducting spoke resonators.

The acceleration cycle to $120 \mathrm{GeV}$ takes 1.4 seconds, so if the linac is operating at $2.5 \mathrm{~Hz}, 5$ out of 7 linac pulses would be available every two cycles for an $8 \mathrm{GeV}$ program, corresponding to $360 \mathrm{~kW}$. Injection is done into the Recycler rather than the Main Injector both to accommodate the slight mismatch between the Main Injector Cycle and the $2.5 \mathrm{~Hz}$ linac rate, and to allow ion stripping and beam restructuring for the $8 \mathrm{GeV}$ program.

Table 1 shows the nominal parameters for Project $X$ and Figure 3 shows where the project would fit relative to other high intensity machines in the world.

In an alternative proposal, beam would be accelerated to slightly more than $2 \mathrm{GeV}$ by a $\mathrm{CW}$ linac. A rapid cycling synchrotron would be used to accelerate beam to $8 \mathrm{GeV}$ for injection into the Recycler. This would leave close to $2 \mathrm{MW}$ of beam power at $2 \mathrm{GeV}$ available for a low energy program. The optimum energy for this beam is being investigated, based on the needs of the Mu2e experiment and potential kaon experiments.

\section{Intermediate Program}

Even in the most optimistic scenario, Project $\mathrm{X}$ will not be complete before late in the next decade, so a series up upgrades has been planned which will support a productive physics program in the intervening years.

Currently, the time it takes to load protons into the Main Injector significantly extend the Main Injector cycle time. After the end of the collider program, a new beam line will allow direct injection from the Booster into the Recycler. The Recycler can then be used to preload protons 
Table 1: Base line parameter table for Project $X$.

\begin{tabular}{|l|rl|}
\hline \multicolumn{2}{|c|}{ Parameter } & Value \\
\hline \multicolumn{2}{|c|}{$\left(H^{-}\right)$} \\
\hline Kinetic Energy & $8.0 \quad \mathrm{GeV}$ \\
\hline Particles per Pulse & $1.6 \times 10^{14}$ \\
\hline Pulse Rate Recycler (protons) \\
\hline Beam Power & $2.5 \mathrm{~Hz}$ \\
\hline \multicolumn{2}{|c|}{$500 \mathrm{~kW}$} \\
\hline Kinetic Energy & $8.0 \mathrm{GeV}$ \\
\hline Cycle Time & 1.4 & $\mathrm{sec}$ \\
\hline Particles/cycle to 120 GeV Program & $1.6 \times 10^{14}$ \\
\hline Particles/cycle to 8 GeV Program & $4.0 \times 10^{14}$ & $($ average) \\
\hline Power to 8 GeV Program & 360 & $\mathrm{~kW}$ \\
\hline \multicolumn{2}{|c|}{ Main Injector (protons) } \\
\hline Kinetic Energy (nominal) & $120.0 \mathrm{GeV}$ \\
\hline Cycle Time & 1.4 & $\mathrm{sec}$ \\
\hline Particles/cycle & $1.6 \times 10^{14}$ \\
\hline Power to 120 GeV Program & $2.1 \quad \mathrm{MW}$ \\
\hline
\end{tabular}

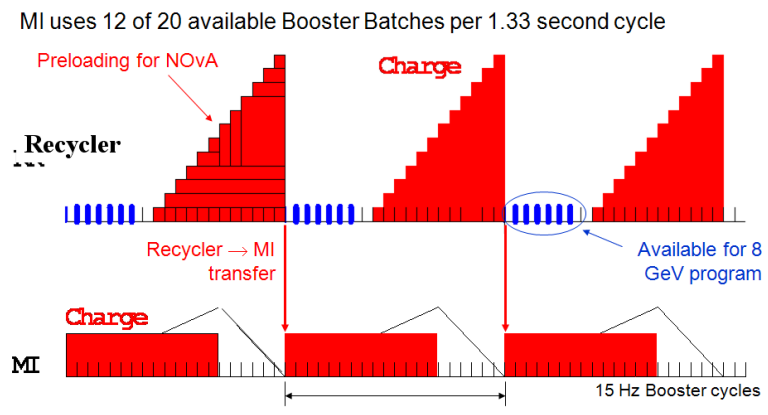

Figure 4: Main Injector capacity limits the total number of protons to the NOvA experiment. This means that a significant number of Booster cycles will be available to the Mu2e experiment during this era.

while the Main Injector is ramping, thereby significantly reducing the cycle time. This will increase the power output of the Main Injector to approximately $700 \mathrm{~kW}$ - about 1/3 of what will be available in the Project X era.

At this point, the total protons available to the $120 \mathrm{GeV}$ program will be limited by the capacity of the Main Injector, leaving additional Booster cycles available to an $8 \mathrm{GeV}$ program, as illustrated in Figure 4. This corresponds to an $8 \mathrm{GeV}$ power of $30 \mathrm{~kW}$, yielding approximately $4 \times 10^{20}$ protons per year.

Unfortunately, the bunch structure produced by the Booster is not well suited to Mu2e or any of the other $8 \mathrm{GeV}$ programs which have been proposed. For the reason, as scheme has been proposed to deliver the protons into the Accumulator and Debuncher, which are currently used for antiprotons. In these rings, the beam will be accumulated, rebunched, and slow extracted to 


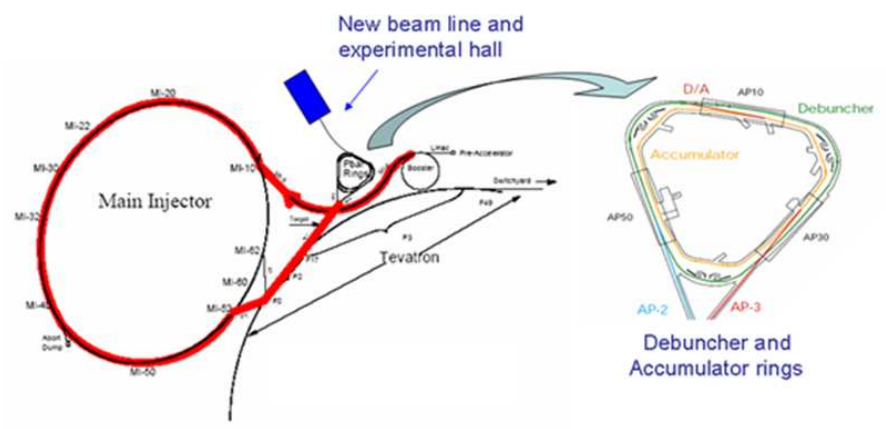

Figure 5: In the so-called "boomerang scheme", protons from the Booster will complete a partial revolution around the Recycler before being transfered to the Accumulator ring. This will allow beam to be sent to the Accumulator with virtually no civil construction.

supply the needs of the Mu2e experiment. To reduce civil construction, beam will be routed to the Accumulator by sending it roughly three quarters of the way around the Recycler, as illustrated in Figure 5.

\section{Conclusion}

In response to the startup of the LHC, Fermilab has produced a plan for a world class physics program based on high intensity proton beams at both low and high energy. The plan is staged, modifying the existing complex to provide valuable physics throughout the next decade while the lab works toward the ultimate goal of program based on a new, high intensity proton source.

\section{References}

[1] "The MINOS Experiment and NuMI Beam Line", http: / / www-numi .fnal .gov/

[2] "BooNE: Booster Neutrino Experiment", http: //www-boone.fnal .gov/

[3] P5 Committee, "US Particle Physics:Scientific Opportunities", http://www.er.doe.gov/hep/files/pdfs/P5_Report\%2006022008.pdf (2008)

[4] "The NOvA Experiment at Fermilab", http://www-nova.fnal .gov/

[5] "Deep Underground Science and Engineering Laboratory", http://www.lbl.gov/nsd/homestake/

[6] "The Mu2e Experiment at Fermilab", http://mu2e.fnal.gov/

[7] Flavor Physics Group, "Consideration of an Experimental Kaon Physics Program at Fermilab in the post Run-II era", http://www.fnal.gov/directorate/Longrange/Steering_Public/files/ (wrap) FutureKaonsV2.0.pdf (2007)

[8] g-2 Collaboration, "The New (g-2) Experiment: A Proposal to Measure the Muon Anomalous Magnetic Moment to \pm 0.14 ppm Precision", http://www.fnal.gov/directorate/program_planning/Mar2009PACPublic/ (wrap) Proposal_g-2-3.0Feb2009.pdf (2009)

[9] "Project X", http://projectx.fnal.gov/ 